\title{
Adaptation of GUARDIAN for Syndromic Surveillance During the NATO Summit
}

\author{
Julio C. Silva ${ }^{1}$, Dino P. Rumoro ${ }^{1}$, Shital C. Shah ${ }^{1}$, Gillian S. Gibbs¹, Marilyn M. Hallock ${ }^{1}$, \\ Michael Waddell ${ }^{2}$ and Shon Doseck ${ }^{2}$
}

${ }^{1}$ Rush University, Chicago, IL, USA; 'Pangaea Information Technologies, Chicago, IL, USA

\section{Objective}

To develop and implement a framework for special event surveillance using GUARDIAN, as well as document lessons learned postevent regarding design challenges and usability.

\section{Introduction}

Special event driven syndromic surveillance is often initiated by public health departments with limited time for development of an automated surveillance framework, which can result in heavy reliance on frontline care providers and potentially miss early signs of emerging trends. To address timelines and reliability issues, automated surveillance system are required.

\section{Methods}

The North Atlantic Treaty Organization (NATO) summit was held in Chicago, IL, May 19-21, 2012. During the NATO summit, the Chicago Department of Public Health (CDPH) was charged with collecting and analyzing syndromic surveillance data from emergency department (ED) visits that may indicate a man-made or naturally occurring infectious disease threat.

Ten days prior to the NATO summit surveillance period, Rush University Medical Center (RUMC) received a guidance document from $\mathrm{CDPH}$ outlining the syndromes for systematic surveillance, specifically febrile rash illness, localized cutaneous lesion, acute febrile respiratory illness, gastrointestinal illness, botulism-like illness, hemorrhagic illness, along with unexplained deaths or severe illness potentially due to infectious disease and cases due to toxins or suspected poisoning. RUMC researchers collected relevant ICD-9 codes for each syndrome category.

GUARDIAN (1), an automated surveillance system, was programmed to scan patient charts and match free text using National Library of Medicine free-text term to unique medical concept, which were further mapped to relevant ICD-9 codes. The baselines were developed using ED patient data from 1/1/2010 to $12 / 31 / 2011$. Statistical references were established for unsmoothed, 24 hour counts (Baseline $=$ Average; Threshold $=+2$ standard deviations).

During the NATO surveillance timeframe (May 13- 26, 2012) automated results with prior reporting period's counts, reference statistics, and charts were electronically sent to $\mathrm{CDPH}$. In addition, ED charge nurses made manual surveillance reports by telephone at least daily. Open lines of communication were maintained between RUMC and $\mathrm{CDPH}$ during the event to discuss potential positive cases. In addition, a post-event debriefing was conducted to document lessons learned.

\section{Results}

The automated GUARDIAN surveillance reports not only provided timely counts of potentially positive cases for each syndrome but also provided trend analysis with baseline measures. The GUARDIAN User Interface was used to explain what data points could trigger positive cases. The Epic system was used to review patient charts, if further explanation was necessary. The observed counts never exceeded +2 standard deviations during the NATO surveillance period for any of the syndromes.

Based on the debriefing meeting between RUMC and CDPH, the top three achievements and lessons learned were as follows:

1. Quick turnaround time ( $\sim 10$ days) from surveillance concept development to automated implementation using GUARDIAN

2. Surveillance data was timely and reliable

3. Additional statistical information was beneficial to put trends in context

4. System may be too sensitive resulting in false alarms and additional investigative burden on public health departments

5. Need for development of user-interfaces with drill down capabilities to patient level data

6. Clinicians don't necessarily utilize exact terminology used in ICD-9 codes which could result in undetected cases.

\section{Conclusions}

This exercise successfully highlights rapid development and implementation of special event driven automated surveillance as well as collaborative approach between front-line entities such as emergency departments, surveillance researchers, and the department of public health. In addition, valuable lessons learned with potential solutions are documented for further refinements of such surveillance activities.

\section{Keywords}

Emergency department; NATO Summit; automated surveillance

\section{Acknowledgments}

GUARDIAN is funded by US Department of Defense, Telemedicine and Advanced Technology Research Center, Award numbers W81XWH-091-0662 and W81XWH-11-1-0711.

\section{References}

J. Silva, D. Rumoro, M. Hallock, S. Shah, G. Gibbs, M. Waddell, K. Thomas, Disease profile development methodology for syndromic surveillance of biological threat agents, Emerging Health Threats Journal, 2011, 4:11129.

*Gillian S. Gibbs

E-mail: Gillian_Gibbs@rush.edu 